\title{
Carbon Nanotubes Flex as Qubits
}

\section{A suspended carbon nanotube coupled to a double quantum dot makes a mechanical oscillator that serves as a qubit.}

\section{By Rachel Berkowitz}

M any of today's quantum computers encode information in fragile quantum states that are difficult to maintain and scale up. A qubit that instead switches between two mechanical states could bring to quantum computing and sensing the high quality and practical manipulability of mechanical oscillators. Fabio Pistolesi, at the French National Center for Scientific Research (CNRS) and the University of Bordeaux, and his colleagues now propose to implement such a qubit in the form of a carbon nanotube whose flexing behavior is determined by the electronic states of two quantum dots [1]. The design could be used to develop quantum devices with large numbers of qubits and long qubit decoherence times.

A mechanical oscillator can be made into a qubit by introducing a controlled deviation from simple harmonic oscillation. This deviation ensures that a driving force can excite only specific energy levels, corresponding to the qubit's two states. In the device proposed by Pistolesi and his colleagues, this deviation would be achieved by coupling the flexural modes of a

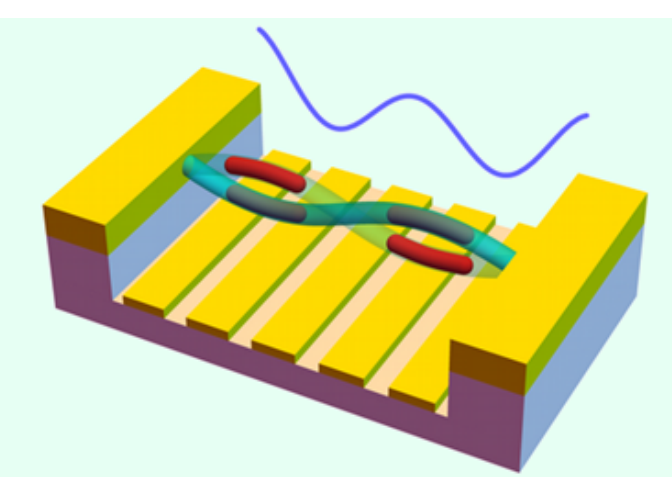

Credit: F. Pistolesi/University of Bordeaux

suspended carbon nanotube to two quantum dots-"artificial atoms" with discrete electronic states-formed in the nanotube itself. The researchers show theoretically that the qubit could be prepared in a given state (one of the nanotube's two lowest oscillation amplitudes) by manipulating the quantum dots' electron localization. The two states could be easily read by a microwave signal. The researchers also show how a logic gate could be implemented to entangle two qubits.

Their robust quantum states make the mechanical oscillators a promising quantum-computing platform. But, the researchers say, because of their sensitivity to classical forces, the oscillators also have potential for detecting faint changes in acceleration, gravity, magnetic moments, and electric forces.

Rachel Berkowitz is a Corresponding Editor for Physics based in Vancouver, Canada.

\section{REFERENCES}

1. F. Pistolesi et al., "Proposal for a nanomechanical qubit," Phys. Rev. X 11, 031027 (2021). 